\title{
Corporate Governance in Non-Profit-Organisationen
}

\section{Verständnisse und Entwicklungsperspektiven}

\author{
Michael MEYER UND FLORENTINE MAIER ${ }^{* *}$
}

Im Rahmen dieses Beitrags wird ein Einstieg in die Diskussion zum Thema Corporate Governance von Non-Profit-Organisationen (NPO) geliefert, indem ein Überblick über unterschiedliche wissenschaftliche und alltagspraktische Verständnisse von Governance gegeben wird. Wissenschaftliche Governanceverständnisse werden charakterisiert, indem die Hauptmerkmale des politikwissenschaftlichen, des betriebswirtschaftlichen und des soziologischen Zugangs zur Governance dargestellt werden. Alltagspraktische Governanceverständnisse werden anhand einer Typologie dargestellt, die von betriebswirtschaftlicher über familiäre, professionalistische und zivilgesellschaftliche bis hin zu basisdemokratischer Governance reicht. Abschließend werden Überlegungen zur Zukunft der Governance von NPO angestellt. Eine weitere Verbreitung des betriebswirtschaftlichen Governance-Diskurses ist wahrscheinlich. Alternative Governance-Zugänge bleiben jedoch notwendige Gegenpole, die wohl in Nischen des Non-Profit-Sektors weiterbestehen werden.

Schlagwörter: Governance, Non-Profit-Organisationen, Diskurs, Managerialismus

\section{Corporate Governance in Nonprofit Organizations: Understandings and Future Perspectives}

This paper opens the discussion about corporate governance of nonprofit organizations by providing an overview of various understandings of governance in research and practice. From the world of research, the major characteristics of political science, management studies, and sociological perspectives on nonprofit governance are drawn out. From nomprofit practice, a typology of everyday notions of governance, including a managerialist, a domestic, a professionalist, a civic, and a grassroots democratic discourse, is presented. The paper closes with reflections about the future of nomprofit governance. A further expansion of managerialist governance discourse seems likely, but alternative notions are expected to survive in niches of the nonprofit sector as necessary counter-poles.

Keywords: Governance, Nonprofit Organizations, Discourse, Managerialism

\footnotetext{
* $\quad$ Beitrag eingereicht am 12.12.2011.

** Univ. Prof. Dr. Michael Meyer, Abteilung für Non-Profit Management/WU Wirtschaftsuniversität Wien, Augasse 2-6, A-1090 Wien, Tel.: +43-(0)1-31336-5366, Fax: +43-(0)1-31336-788, E-Mail: michael.meyer@wu.ac.at, Forschungsschwerpunkte: Organisationsanalyse, Karrieren, Funktionen von Non-Profit-Organisationen, Diffusion betriebswirtschaftlicher Methoden in NPO.

Dr. Florentine Maier, Abteilung für Non-Profit Management/WU Wirtschaftsuniversität Wien, Augasse 2-6, A-1090 Wien, Tel.: +43-(0)1-31336-5896, Fax: +43-(0)1-31336-788, E-Mail: florentine.maier@wu.ac.at, Forschungsschwerpunkte: Rationalisierung und Verbetriebswirtschaftlichung von NPO.
} 


\section{Einleitung}

Der Begriff „Corporate Governance in Non-Profit-Organisationen“ mag auf den ersten Blick als Widerspruch in sich erscheinen, spielen Non-Profit-Organisationen (NPO) in unserer Gesellschaft doch auch die Rolle eines Gegenpols zur „corporate world“. Versteht man unter „Corporate Governance“ jedoch jene Form der Governance, die sich mit der Lenkung und Regulierung einer Organisation beschäftigt, also organisationale oder ,interne“ Governance (Steen-Johnsen et al. 2011), so liegt auf der Hand, dass es sich um ein Thema von hoher Relevanz für NPO handelt. Das Konzept der Governance betont im Gegensatz zu Konzepten wie Management oder Controlling, bei denen es ebenfalls um Steuerung geht, vor allem den Aspekt der Rechenschaftspflicht (siehe etwa Stone/Ostrower 2007) und des Schutzes von Stakeholderinteressen (siehe etwa Speckbacher 2008). Für NPO, deren Handlungsfähigkeit und Überleben in besonderem Maße von der ihnen zugestandenen Legitimität abhängen, ist daher interne Governance unverzichtbar. Dies betrifft insbesondere jene NPO, die sich durch Spenden finanzieren und auf Freiwilligenarbeit aufbauen.

Ziel des vorliegenden Beitrags ist es, einen Einstieg in die Diskussion rund um das Thema Corporate Governance von NPO zu liefern, indem ein Überblick über unterschiedliche wissenschaftliche und alltagspraktische Verständnisse von Governance gegeben wird und Überlegungen über zukünftige Entwicklungen der Nonprofit Governance angestellt werden.

\section{Wissenschaftliche Verständnisse von Governance}

„Governance“ ist einer jener sozialwissenschaftlichen Fachbegriffe, die plötzlich auftauchen, sehr vage bleiben und Bedeutungen in sich tragen, die sich nur durch extensives Studium erschließen (Hugues 2010: 87). Im Rahmen dieses Abschnitts soll daher versucht werden, einen Überblick über die wichtigsten unterschiedlichen Verständnisse von Governance zu geben. Als Eckpunkte für unsere Darstellung wollen wir von den unterschiedlichen sozialwissenschaftlichen Disziplinen ausgehen, die sich mit dem Thema Governance beschäftigen. Dies sind vor allem die Politikwissenschaft, die Betriebswirtschaftslehre und die Soziologie.

Die politikwissenschaftliche Forschung richtet den Fokus auf jene Formen der Governance, durch die ganze Gesellschaften gesteuert werden, die also aus Perspektive einzelner NPO „extern“ sind (Steen-Johnsen et al. 2011; siehe auch Rhodes 1997; Bozzini/Enjolras 2011). Diese Form der Makro-Governance umfasst z. B. Gesetze, Gerichtsentscheidungen, administrativen Regelungen, aber auch ganz allgemein alle Praktiken, die bestimmen, welche und wie öffentliche finanzierte Güter in einer Gesellschaft erstellt werden, und wie sie verteilt werden (vgl. Lynn Jr. et al. 2000: 4). Der Prozess der Makro-Governance wird zunehmend weniger vom Staat gesteuert. Stattdessen gewinnen marktwirtschaftliche Akteure und Steuerungsprinzipien an Bedeutung sowie, in weitaus bescheidenerem Rahmen, auch NPO, die mit der Implementierung öffentlicher Aufgaben beauftragt werden. Governance wurde und wird in der politikwissenschaftlichen Diskussion als Alternativ- und Gegenbegriff zu Government aufgebaut (Rosenau/Czempiel 1992).

Die Betriebswirtschaftslehre beschäftigt sich mit Fragen der Corporate Governance, also der internen, organisationalen Governance. Zielsetzung ist hierbei meist auch, 
normative Gestaltungsempfehlungen abzugeben. Eine Reihe betriebswirtschaftlicher Theorien eröffnet unterschiedliche Sichtweisen auf das Phänomen Governance (vgl. Cornforth 2003).

Für die Prinzipal-Agent-Theorie (Jensen/Meckling 1976) geht es bei Governance darum, wie die Prinzipale einer Organisation sicherstellen können, dass ihre Interessen von den Agenten (i. d. R. den angestellten ManagerInnen) gewahrt werden. Es wird davon ausgegangen, dass auch in NPO unterschiedliche Akteure verschiedene Interessen haben, und dass das Management durchaus Eigeninteressen oder zumindest seine eigene Interpretation der Mission der NPO verfolgt. Wichtigstes Instrument der Governance ist aus dieser Perspektive das Kontrollorgan (der „Aufsichtsrat") der NPO. In diesem sollen die Prinzipale vertreten sein, um die Aktivitäten des Managements zu überwachen. Betreffend der Frage, wer denn die Prinzipale einer NPO sein sollen, schlägt Speckbacher (2008) vor, jene Akteure zu wählen, die spezifische Beiträge leisten, für die die Gegenleistung nicht durch Verträge gesichert ist. Dies können von Fall zu Fall SpenderInnen und StifterInnen (kritisch dazu siehe Benjamin 2010), Vereinsmitglieder, Freiwillige oder MitarbeiterInnen, die mehr als das arbeitsvertraglich Abgedeckte leisten, sein. Es wird angeraten, nicht übermäßig viele unterschiedliche Akteursgruppen ins Aufsichtsorgan aufzunehmen, da diese auch die Kosten der Entscheidungsfindung erhöhen (Speckbacher 2008).

Ein Plädoyer für eine breitere Berücksichtigung von Akteuren in der Nonprofit Governance lässt sich aus der Stakeholder Theorie nach Freeman (1984) ableiten. Freeman sieht als Stakeholder nicht nur jene Akteure, die wertvolle spezifische Ressourcen für die Organisation liefern, sondern alle, die von den Aktivitäten einer Organisation betroffen sind oder auf diese Einfluss nehmen können. Das inkludiert im Gegensatz zur Prinzipal-Agent-Theorie zum Beispiel auch KlientInnen und andere NPO (die etwa durch Spendenskandale in Mitleidenschaft gezogen werden können). Die Stakeholder Theorie empfiehlt, dass NPO ein breites Spektrum unterschiedlicher Stakeholder in ihre Governance involvieren sollen, um unterschiedlichen Umweltansprüchen gerecht zu werden. Das Aufsichts- bzw. Leitungsorgan einer NPO fungiert aus dieser Perspektive vor allem als Forum, wo widersprüchliche Interessen integriert und organisationale Ziele und Richtlinien ausgehandelt werden (Cornforth 2003).

Eine weitere Alternative zur Prinzipal-Agent-Theorie stellt die Stewardship-Theorie (Muth/Donaldson 1998) dar. Hier wird auf der Basis eines diametral entgegengesetzten Menschenbildes angenommen, dass ManagerInnen nicht ihre Eigeninteressen verfolgen, sondern vielmehr versuchen, treue Hüter der Ressourcen und der Mission der Organisation zu sein. Aus dieser Perspektive sollten das Management und andere Stakeholder in den Aufsichts- bzw. Leitungsgremien partnerschaftlich zusammenarbeiten. Aufgabe dieser Gremien ist es nicht, das Management zu kontrollieren, sondern die Leistung der Organisation zu steigern. Dazu wird empfohlen, Personen mit nützlichen Qualifikationen oder Kontakten in diese Gremien aufzunehmen und Teambildungsmaßnahmen zu betreiben (Cornforth 2003).

Eine weitere wichtige betriebswirtschaftliche Theorie, die Themen der organisationalen Governance berührt, ist die Resource-Dependency-Theorie (Pfeffer/Salancik 2003). Diese geht von der Idee aus, dass jede Organisation für ihr Überleben Ressour- 
cen aus ihrer Umwelt braucht. Den Leitungs- und Aufsichtsorganen kommt aus dieser Perspektive eine Brückenfunktion zu. Sie sollen die Ressourcenabhängigkeiten der Organisation handhaben, etwa indem sie der Organisation wichtige Informationen zutragen, Zugang zu materiellen Ressourcen sichern und das öffentliche Image der Organisation verbessern (Miller-Millesen 2003). Auch die Kooptierung externer Akteure durch Mitgliedschaft im Aufsichtsorgan gehört zu den Gestaltungsempfehlungen, die sich aus dieser Theorie ableiten lassen (Cornforth 2003).

Gemeinsam ist der betriebswirtschaftlich orientierten Forschung ein starker Fokus auf die Leitungs- und Aufsichtsorgane von NPO. Erst in jüngster Zeit wurde die Forderung lauter, das ganze Governance-System von NPO in den Blick zu nehmen, also beispielsweise auch Audits, Berichtspflichten, Inspektionen und gesetzliche Auflagen (Cornforth 2011).

Eine umfassendere Sichtweise von organisationalen Governance-Systemen findet sich bislang am ehesten in der soziologisch ausgerichteten Forschung zum Thema. So zählt etwa Enjolras (2009) zum Governance-System nicht nur die Verteilung von Residualrechten, Kontroll- und Rechenschaftsmechanismen sowie Entscheidungsverfahren, sondern auch formale Organisationsziele, Eigentumsrechte und die durch diese Elemente entstehenden Anreize für das Management. Sofern solche Studien überhaupt normativ argumentieren, treten sie typischerweise besonders für demokratische und partizipative Governance-Strukturen ein (siehe z. B. Enjolras 2009; LeRoux 2009).

Viele (organisations-)soziologisch ausgerichtete Studien versuchen zu erklären, warum in NPO so unterschiedliche Governance-Systeme eingesetzt werden. Hierbei werden meist Erklärungsansätze aus unterschiedlichen Theorien eklektisch herangezogen (siehe z. B. Alexander/Weiner 1998; Miller-Millesen 2003; Bradshaw 2009; Ostrower/Stone 2010). In den hierbei entwickelten Erklärungsrahmen wird typischerweise zwischen internen und externen Einflussfaktoren auf organisationale Governance-Systeme unterschieden. Zu den internen Einflussfaktoren zählen etwa das Alter, die Größe, der Professionalisierungsgrad, die Ressourcenausstattung, der Stabilitätsgrad, die ideologische Ausrichtung und die Strategie der NPO. Zu den externen Einflussfaktoren zählen vor allem die institutionellen Normen im Feld, vermittelt durch eventuelle Muttergesellschaften, Geldgeber, Aufsichtsbehörden und rechtliche Regelungen.

In jüngster Zeit wird vermehrt gefordert, Themen der Makro-Governance und der organisationalen Governance in Verbindung zu betrachten (Stone/Ostrower 2007; Cornforth 2011). Relevant für dieses Unterfangen erscheint insbesondere das Foucaultsche Konzept der Gouvernementalität. Es bezeichnet eine Gesamtheit von Institutionen, Verfahren, Analysen, Reflexionen, Berechnungen und Taktiken zur Ausübung von Macht. Ihre Zielscheibe ist die Bevölkerung, ihre Hauptwissensform ist die politische Ökonomie, und ihr wesentliches technisches Instrument sind die Sicherheitsdispositive (Foucault 2000: 64). Governementalität bedeutet Regieren durch Selbststeuerung in Form komplexer Wirkungsketten; politische Macht dringt über kapillare Verzweigungen (die auch NPO einschließen) bis in den Kern der einzelnen Individuen vor (Foucault 2007 [1975]: 68). Exemplarisch für eine Anwendung dieser Perspektive ist etwa Seckinelgins (2004) Analyse der Governance von HIV/AIDS im subsaharischen Afrika: Von WHO, Weltbank, IMF, der Afrikanischen Union, der EU, individuellen Geberländern und ihre Agenturen, über internationale NGOs und Unternehmen sowie die Regierungen der betroffenen Länder, zu lokalen 
NPO und schließlich den einzelnen betroffenen Menschen spannt sich das Netz der Governance. Seckinelgin skizziert ein Governance-System, in dem die politische Macht im wesentlichen von „oben“ nach „unten“ fließt und der Handlungsspielraum von lokalen NPO durch mächtigere Akteure stark eingeschränkt wird. Einen anderen Aspekt der Rolle von NPO in Systemen der Gouvernementalität beleuchtet Villadsen (2009): Er weist darauf hin, das NPO selbst eine entscheidende Rolle bei der Konstruktion von interventionsbedürftigen Bevölkerungsgruppen und geeigneten Interventionstechniken spielen. Diese Konstruktionen werden dann oft später in den $\mathrm{Zu}$ ständigkeitsbereich staatlicher Politik übernommen (z. B. im Bereich der Drogentherapie).

Nach diesem Überblick über unterschiedliche theoretische Konzeptionen von Governance in verschiedenen Disziplinen sollen nun die alltagspraktischen Verständnisse der Governance in NPO beleuchtet werden.

\section{Alltagspraktische Verständnisse der organisationalen Governance von NPO}

Versteht man unter einem organisationalen Governance-System die Gesamtheit aller steuerungswirksamen Strukturen in einer Organisation, welche die Erfüllung von Rechenschaftspflichten und den Schutz von Stakeholderinteressen sicherstellen, so liegt auf der Hand, dass praktisch jede NPO über irgendeine Form von GovernanceSystem verfügt. Dieses mag die Stakeholderinteressen mehr oder weniger gut vertreten und die Gestaltungsempfehlungen, die aus unterschiedlichen theoretischen Perspektiven abgeleitet wurden, mehr oder weniger berücksichtigen.

In diesem Abschnitt sollen die in der Praxis existierenden Verständnisse von Nonprofit-Governance dargestellt werden. Wir berichten dazu aus Ergebnissen eines empirischen Forschungsprojekts, welches wir 2008/2009 mit NPO in Österreich durchgeführt haben. Details zur Methode sowie weitere Ergebnisse finden sich bei Maier und Meyer (2011). Im Rahmen dieses Forschungsprojekts wurden qualitative Interviews mit VertreterInnen von NPO geführt, um mehr über ihr Verständnis von Organisation und Governance zu erfahren. Mittels diskursanalytischer Verfahren wurde dann eine Typologie von Diskursen des Organisierens entwickelt und auf ihre Implikationen für die Governance von NPO hin untersucht.

Es zeigt sich, dass bei den alltagspraktischen Verständnissen von organisationaler Governance in NPO fünf Typen unterschieden werden können: (1) „managerialistische“, (2) „familiäre“, (3) „professionalistische“, (4) „,bürgerschaftliche“ und (5) „basisdemokratische" Governance (siehe die Übersicht in Tabelle 1). Wir orientieren uns für die Darstellung dieser Typen an drei Fragen zur Rechenschaftspflicht von NPO, die durch Governance erfüllt werden soll (vgl. Stone/Ostrower 2007): „Wem gegenüber ist die Organisation rechenschaftspflichtig?“, „Wie kann die Rechenschaftspflicht erfüllt werden?“ und: „Was sind Kriterien für die Leistung, für welche die Organisation rechenschaftspflichtig ist?“ 


\begin{tabular}{|c|c|c|c|c|c|}
\hline & $\begin{array}{l}\text { Manageria- } \\
\text { listisch }\end{array}$ & Familiär & $\begin{array}{l}\text { Professiona- } \\
\text { listisch }\end{array}$ & $\begin{array}{c}\text { Bürgerschaft- } \\
\text { lich }\end{array}$ & $\begin{array}{c}\text { Basisdemo- } \\
\text { kratisch }\end{array}$ \\
\hline $\begin{array}{l}\text { Adressaten der } \\
\text { Governance }\end{array}$ & GeldgeberInnen & $\begin{array}{l}\text { Leistungs- } \\
\text { empfängerInnen }\end{array}$ & $\begin{array}{l}\text { Externe Fach- } \\
\text { kollegInnen }\end{array}$ & $\begin{array}{c}\text { Aktive } \\
\text { Mitglieder }\end{array}$ & AktivistInnen \\
\hline $\begin{array}{l}\text { Governance- } \\
\text { Mechanismen }\end{array}$ & $\begin{array}{c}\text { Aufsichtsorgan, } \\
\text { Geschäfts- } \\
\text { führung }\end{array}$ & $\begin{array}{c}\text { Persönliche } \\
\text { Beziehungen, } \\
\text { Gefühle }\end{array}$ & $\begin{array}{l}\text { Bewertung } \\
\text { durch Fachkol- } \\
\text { legInnen, Ver- } \\
\text { gleich mit ande- } \\
\text { ren Organisati- } \\
\text { onen im Feld }\end{array}$ & $\begin{array}{l}\text { Wahlen, Ab- } \\
\text { stimmungen, } \\
\text { gegenseitige } \\
\text { Kontrolle, } \\
\text { Einhalten for- } \\
\text { maler Regeln }\end{array}$ & $\begin{array}{l}\text { Herrschaftsfreie } \\
\text { Diskussion, } \\
\text { Konsenssuche, } \\
\text { Offenheit der } \\
\text { Organisation }\end{array}$ \\
\hline $\begin{array}{l}\text { Leistungs- } \\
\text { kriterien }\end{array}$ & $\begin{array}{l}\text { Effektives und } \\
\text { effizientes } \\
\text { Erreichen einer } \\
\text { expliziten } \\
\text { Mission }\end{array}$ & $\begin{array}{c}\text { Erreichen } \\
\text { einer impliziten } \\
\text { Mission }\end{array}$ & $\begin{array}{l}\text { Einhaltung } \\
\text { professioneller } \\
\text { Standards, } \\
\text { positive Bewer- } \\
\text { tung durch } \\
\text { FachkollegInnen }\end{array}$ & $\begin{array}{c}\text { Breite } \\
\text { Unterstützung }\end{array}$ & $\begin{array}{l}\text { Einhalten von } \\
\text { Regeln der } \\
\text { Basisdemokratie }\end{array}$ \\
\hline
\end{tabular}

\section{Tabelle 1: Alltagspraktische Verständnisse von organisationaler Governance in NPO (Quelle: Maier/Meyer 2011)}

(1) Im managerialistischen Verständnis von organisationaler Governance sind die SpenderInnen und Geldgeber-Institutionen die primären Adressaten von organisationaler Governance, denn ihre Rolle wird als den Eigentümern in gewinnorientierten Unternehmen am ähnlichsten gesehen. Wichtigster Governance-Mechanismus ist das Zusammenspiel zwischen dem Aufsichtsorgan der NPO („Aufsichtsrat" ) und der Geschäftsführung. Es wird angenommen, dass das Aufsichtsorgan für strategische Weichenstellungen und die Überwachung der Geschäftsführung zuständig ist, während die Geschäftsführung freie Hand in operativen Fragen haben soll. Referenzpunkt für die Rechenschaftspflicht ist die schriftlich festgehaltene Mission der NPO bzw. sind es die aus ihr abgeleiteten Ziele, welche effektiv und unter effizientem Mitteleinsatz erreicht werden sollen.

(2) Ganz anders stellt sich das „familiäre“ Verständnis von Governance dar. Hier werden die LeistungsempfängerInnen als primäre Adressaten von Governance angesehen. Es wird angenommen, dass gerade gegenüber den Schwächsten und Bedürftigsten die größte Rechenschaftspflicht besteht. Zur Umsetzung dieses Rechenschaftsanspruchs wird auf paternalistische und informelle Methoden zurückgegriffen; die direkte Mitwirkung der LeistungsempfängerInnen an Governance-Prozessen ist nicht vorgesehen. Vielmehr sollen die ErstellerInnen der Leistung die Bedürfnisse der Leistungsempfänger im persönlichen Kontakt erfahren, empathisch verinnerlichen, und sich in ihrem Handeln davon leiten lassen. Diese Governance-Mechanismen basieren also auf Gefühlen (Empathie) und persönlichen Beziehungen. Auch werden Leistungskriterien nicht explizit festgelegt, geschweige denn formal überprüft. Vielmehr teilen die Mitglieder der Organisation ein implizites Verständnis vom Ziel und von Leistungsnormen. 
Für die akademische Diskussion zum Thema Governance stellt das familiäre Governance-Verständnis typischerweise eine Art von Gegenfolie im Sinne einer „NichtGovernance“ oder „schlechten Governance“ dar. Tatsächlich sind GovernanceSkandale in der NPO-Praxis meist auf unangemessenes Vertrauen in informelle, persönliche, „gefühlsbasierte“ Governance-Mechanismen zurückzuführen. Allerdings muss zugestanden werden, dass es sich um ein einfaches und für viele Menschen unmittelbar einleuchtendes Governance-Verständnis handelt, das in kleinen oder jungen NPO durchaus seine Berechtigung hat.

(3) Ein weiteres in der Praxis bedeutsames Governance-Verständnis kann als „professionalistisch“ charakterisiert werden. Elemente des professionalistischen Governance-Verständnisses finden sich oft bei NPO, die in Bereichen der Wissenschaft, Medizin oder Pädagogik tätig sind, aber auch z. B. in von Freiwilligenarbeit geprägten Bereichen wie Sport oder Feuerwehr. Hier werden die Normen und Standards der Profession bzw. des Fachs als zentrale Referenz angesehen. Primäre Adressaten der Governance sind daher organisationsexterne FachkollegInnen, die diese Standards repräsentieren. FachkollegInnen überprüfen die Konformität mit professionellen Standards, entweder durch unmittelbare Bewertung der Organisation anhand dieser Standards, oder durch Vergleiche von Organisationen innerhalb des Feldes. Beispiele unmittelbarer Bewertungen sind etwa die Inspektion durch Aufsichtsbehörden oder die Akkreditierung durch Akkreditierungsinstitutionen. Beispiele für Vergleiche sind etwa die Teilnahme an Wettbewerben oder das mehr oder weniger strukturierte Mitverfolgen der Aktivitäten anderer Organisationen im Feld. Professionalistisch ausgerichtete NPO erbringen typischerweise hochkomplexe Leistungen, deren Qualität für Fachfremde nicht unmittelbar transparent ist und bei denen eine Steuerung über reine Outputkriterien zu unerwünschten Effekten führen kann. Als die sinnvollsten Kriterien für gute Leistung werden in diesen Fällen daher die Einhaltung professioneller Standards und die positive Bewertung durch FachkollegInnen angesehen.

(4) Im bürgerschaftlichen Governance-Verständnis wird von einer mitgliedschaftlich organisierten NPO, also einem Verein oder einer Genossenschaft, ausgegangen. Hier stehen demokratische Mitbestimmungsrechte im Mittelpunkt der Überlegungen. Es wird angenommen, dass die primäre Rechenschaftspflicht gegenüber den aktiven Mitgliedern besteht. Dementsprechend sind Wahlen und Abstimmungen wichtige Governance-Mechanismen. Auch ein ausgeklügeltes System von Gremien, die sich gegenseitig kontrollieren, sowie ein umfangreiches formales Regelwerk, dessen Einhaltung kontrolliert wird, tragen zur Governance bei. Es wird angenommen, dass gute Leistungserfüllung am besten darin erkennbar ist, dass die NPO sich breiter Unterstützung erfreut. Als Leistungskriterien dienen daher zum Beispiel hohe Mitgliederzahlen, eine starke Durchdringung des Wirkungsbereiches (z. B. bei Gewerkschaften ein hoher Anteil gewerkschaftlich organisierter Betriebe) oder (im Fall von politischen Parteien) Wahlgewinne.

(5) Als letztes praktisch bedeutsames Governance-Verständnis konnte das basisdemokratische identifiziert werden. Bei diesem, welches unter anderem auf Ideen der 1968er Bewegung aufbaut, stehen Ideale der Autonomie und des konsensualen Entscheidens im Mittelpunkt. Die Rechenschaftspflicht wird primär gegenüber jenen Mitgliedern der Organisation angenommen, deren wesentlicher Beitrag ihre Mitarbeit 
ist. Sie müssen alle Entscheidungen der Organisation tragen. Jedes Mitglied hat das Recht, alle organisationalen Entscheidungen in Frage zu stellen. Gleichzeitig versucht die Organisation, externe Abhängigkeiten, etwa gegenüber Geldgebern, möglichst gering zu halten. Um die Einhaltung der Rechenschaftspflicht gegenüber ihren aktiven Mitgliedern zu sichern, gibt es ein breites Repertoire basisdemokratischer Governance-Mechanismen, vor allem Regeln für herrschaftsfreies Diskutieren (z. B. Formulierregeln, RednerInnenlisten, Redezeitquoten) und verschiedene Verfahren der Konsenssuche. Ein weiterer wichtiger Governance-Mechanismus ist das Prinzip der organisationalen Offenheit: Alle, die sich für die Themen der Organisation interessieren, sind zum Mitreden eingeladen und auch dazu, ihre kritische Sichtweise einzubringen. Weil basisdemokratische Organisationen oft radikale gesellschaftliche Veränderungen anstreben, die sich wenn überhaupt nur sehr langsam einstellen, wäre für diese Organisationen Zielerreichung kein praktikables Leistungskriterium. Als Leistungskriterium dient vielmehr die Einhaltung basisdemokratischer Prinzipien: Es wird als Erfolg angesehen, dass die Organisation die Möglichkeit basisdemokratischer Zusammenarbeit an Ort und Stelle demonstriert. In Reinform findet sich dieses basisdemokratische Verständnis von Governance in Nischen des Nonprofit-Sektors im links-alternativen Bereich (in Österreich etwa bei den „Grünen und Alternativen StudentInnen“ oder dem Fahrradbotendienst „Hermes“). Breiteres Legitimations- und Mobilisierungspotenzial hat dieses Governance-Verständnis aktuell weniger in Organisationen sondern eher in sozialen Bewegungen, etwa bei den Aufständen in Tunesien und Ägypten, der Occupy-Bewegung, oder - um ein österreichisches Beispiel zu nennen - der „Unibrennt"-Bewegung.

Bei Betrachtung dieser unterschiedlichen Governance-Verständnisse wird deutlich, dass bei der Gestaltung von Governance-Systemen in NPO höchst unterschiedliche normative Annahmen zugrunde gelegt werden können. Im abschließenden Teil dieses Beitrags wollen wir uns der Frage zuwenden, welche Trends bei diesen normativen Annahmen zu beobachten sind, und welche zukünftigen Entwicklungen wahrscheinlich erscheinen.

\section{Trends und Zukunftsperspektiven der Governance von NPO}

Gesellschaftliche Sichtweisen, was „gute Governance“ sei, haben seit den 1980er Jahren einen offensichtlichen Wandel durchlaufen. Allein die Verbreitung des Begriffes und der damit verbundene Übergang von einem engeren kontrollorientierten zu einem weiteren steuerungsorientierten Verständnis sozialer Systeme verdeutlichen diesen Wandel. Wir vermuten hinter diesen Entwicklungen im Bereich der organisationalen und gesellschaftlichen Governance mehrere Einflussfaktoren.

Auf gesellschaftlicher Ebene lässt sich die Entwicklungstendenz treffend mit dem Konzept der „Post-Demokratie“ (Crouch 2004) charakterisieren. Governance-Systeme beziehen ihre Legitimität heute längst nicht mehr vorrangig aus der Partizipation der Betroffenen mittels demokratischer Verfahren. Ihre Legitimität schöpft sich vielmehr primär aus der Fähigkeit, weithin als wünschenswert angesehene Resultate zu produzieren. Ein solches System kann partizipative Verfahren beinhalten, aber nur, wenn sie anderen Verfahren an Effizienz und Effektivität überlegen sind. So kommt es auch, dass der Zivilgesellschaft und damit den NPO eine wichtige Rolle in der gesellschaftlichen Go- 
vernance zugeschrieben wird. Mit einer Stärkung der Zivilgesellschaft ist immer die Hoffnung verbunden gewesen, dass damit partizipative Elemente in der gesellschaftlichen Governance gestärkt werden, ohne substantielle Effizienz- und Effektivitätsverluste einzufahren (vgl. z. B. das Forschungsnetzwerk CINEFOGO - Civil Society and New Forms of Governance, www.cinefogo.com, weiters z. B. Zimmer/Freise 2007; Evans et al. 2005; Stone/Ostrower 2007). Kaum berücksichtigt wird dabei aber, dass die demokratische Legitimation vieler NPO durchaus umstritten ist.

Der Stellenwert der internen Governance hatte bislang erstaunlich wenige Anknüpfungspunkte an die Rolle von NPO in der Zivilgesellschaft bzw. in der gesellschaftlichen Governance. Das Argument, dass NPO besondere Ansprüche an ihre organisationale Governance stellen müssen, wenn sie selbst an der gesellschaftlichen Steuerung partizipieren wollen, wurde bislang kaum gehört. Was die Nonprofit-Governance betrifft, so zeigt sich heute eher eine verzögerte Replikation des auf Effizienz verengten Corporate-Governance-Diskurses. Das war nicht immer so. Von den späten 1960ern bis in die späten 1980er war es im Nonprofit-Sektor durchaus legitim, demokratischer Beteiligung den Vorrang gegenüber Effizienzüberlegungen einzuräumen. Ein Mindestmaß an ökonomischer Effizienz wurde zwar als notwendige Voraussetzung angesehen, aber das Einhalten demokratischer Prozeduren galt als klares Kriterium für „good governance“, ohne dass damals irgendjemand diese Begrifflichkeit verwendet hätte. Die „Demokratisierung aller Lebensbereiche“ sollte die Menschen glücklich machen und ihnen ermöglichen, sich selbst zu verwirklichen. Diese Philosophie beseelte in der westlichen Welt zahlreiche Initiativen im Kultur- und Sozialbereich, sowie im Bereich der alternativen Ökonomie.

Die Zeiten haben sich aber geändert. Heute wird in den meisten Publikationen zur Nonprofit-Governance geschrieben, dass es der Hauptzweck organisationaler Governance-Systeme sei, die effiziente Missionserreichung sicherzustellen. Demokratie sei nur insofern wertvoll, als sie dazu einen Betrag leistet. Grundsätzlich sollen aber Governance-Systeme die Kosten der Entscheidungsfindung möglichst niedrig halten. Somit wurden zuerst einmal der basisdemokratische und der bürgerschaftliche Diskurs der Governance in den Hintergrund gedrängt. Der familiäre Diskurs war ohnehin über weite Strecken als „unprofessionell“ diskreditiert; lediglich das professionalistische Verständnis von Governance leistet in bestimmten Bereichen, etwa im Gesundheits- und im Erziehungssystem, noch hinhaltenden Widerstand.

Wir vermuten, dass dieser Trend anhalten wird. NPO werden fortfahren, ihre Governance-Systeme in Hinblick auf effizientere Leistungserstellung weiterzuentwickeln, was in vielen Fällen einen Abschied von demokratischen Elementen dieser Systeme bedeuten wird. Die Präferenz für Effizienz gegenüber Partizipation scheint einen breiten gesellschaftlichen Konsens darzustellen. Eine Revolution von unten, in der exkludierte Stakeholder mehr Einfluss über ,ihre“ NPO fordern würden, ist derzeit nicht in Sicht. KleinspenderInnen, Mitglieder, und auch LeistungsempfängerInnen scheinen weitestgehend damit zufrieden, ihre Rolle als KonsumentInnen anzulegen, die zwischen verschiedenen verfügbaren Alternativen wählen und ihre Einflussnahme auf die Exit-Option beschränken. Die Entscheidungsmacht wird ManagerInnen und sonstigen ExpertInnen in den NPO überlassen. Hintergrund dieser Entwicklung mag sein, dass die Mehrzahl der Menschen bis an ihre Grenzen damit ausgelastet ist, die 
anwachsenden Risiken, Chancen und Verantwortlichkeiten in ihrem Leben zu bewältigen. Von ManagerInnen, auch in NPO, wird da erwartet, Leadership zu zeigen und die Versorgung mit wichtigen Dienstleistungen und Produkten sicherzustellen (Blühdorn 2007).

Die Corporate Governance für gewinnorientierte Aktiengesellschaften, wie sie im Gefolge des Enron-Skandales 2001 propagiert wurde, bildet also den Hauptbezugspunkt für die Empfehlungen zur Nonprofit-Governance. Sie sollen sicherstellen, dass die Mission der NPO erfüllt wird und zentrale Stakeholderinteressen nicht gefährdet werden. Zwar werden dann die Besonderheiten der Nonprofit-Governance ausführlich diskutiert, also bspw. die ausdifferenzierte und je nach Organisation unterschiedliche Konstellation der schützenswerten Interessen von Stakeholdern und die Schwierigkeit bei der Operationalisierung der Mission (Budäus 2005; Siebart 2006; von Schnurbein 2008). Die besondere gesellschaftliche Bedeutung von NPO spielt dabei aber keine Rolle.

Auf einer Art Meta-Ebene lässt sich zunehmend ein professionalistischer Diskurs der managerialistischen Governance beobachten: Bei der Implementierung von Governance-Systemen verlassen sich NPO weniger auf ihre eigenen Kompetenzen als vielmehr auf externe Standards und die Unterstützung von Beratungsunternehmen. Dies zeigt sich in einer Tendenz zur Kodifizierung der Corporate Governance managerialistischer Prägung. Sowohl für Deutschland als auch für die Schweiz liegen zahlreiche derartige Kodizes vor (von Schnurbein/Stöckli 2010). Damit zeigen sich Prozesse der Isomorphie auch im Bereich der Nonprofit-Governance, wie üblich angetrieben durch Beratungsunternehmen, Zertifizierungsagenturen und NPO-Mitglieder mit betriebswirtschaftlichem Ausbildungs- oder Berufshintergrund (vgl. Hwang/Powell 2009).

Dennoch wäre es verfrüht und falsch zu behaupten, dass post-demokratische Entwicklungen die partizipative Governance vollkommen verdrängen. Organisationen entwickeln laufend neue Formen der Partizipation, die weniger Zeit und Energie erfordern. Offizielle Bekenntnisse zum Wert der Partizipation gehören weiterhin zum ideologischen Repertoire der meisten modernen NPO. Diese Partizipation ist jedoch, wie oben ausgeführt, nicht mehr ein Wert an sich. Sie erfüllt vielmehr technischinstrumentelle Zwecke oder dient der symbolischen Legitimation der Organisation.

Aufmerksamen BeobachterInnen des Nonprofit-Sektors wird es nicht schwer fallen, Einzelbeispiele zu finden, die dieser Perspektive zur Zukunft der NonprofitGovernance widersprechen. Dies führt uns zu unserer abschließenden Vermutung: Es darf erwartet werden, dass alternative Sichtweisen über den Zweck und die richtige Ausgestaltung von Governance-Systemen auch weiterhin in gewissen Nischen überleben und praktiziert werden. Dies betrifft nicht nur das besonders demokratie-affine bürgerschaftliche und basisdemokratische Governance-Verständnis, sondern auch das professionalistische und das familiäre. Diese werden vorerst als Abweichungen von der Norm toleriert, und zwar durchaus im Sinn der Verstärkung des herrschenden, des managerialistischen, Diskurses: Immer wieder werden die vorhandenen Auswüchse anderer Governanceformen - das Fehlverhalten Einzelner und der Vertrauensverlust bei familiärer Governance, die Selbstlähmung der Entscheidungsprozesse bei demokratischer Governance und die Überstandardisierung und der Autonomieverlust bei 
professionalistischer Governance - als argumentative Pappkameraden aufgebaut und mit den überlegenen Geschützen managerialistischer Governance umgeschossen. Dies entspricht der Foucaultschen Annahme über den strikt relationalen Charakter der Machtverhältnisse. Machtverhältnisse „können nur kraft einer Vielfalt von Widerstandspunkten existieren, die in den Machtbeziehungen die Rolle von Gegnern, Zielscheiben, Stützpunkten, Einfallstoren spielen“ (Foucault 1983: 96). Das managerialistische Governance-Verständnis braucht also für seinen Fortbestand alternative Governance-Verständnisse, von denen es sich als die „bessere“ Form der Governance abgrenzen kann.

\section{Literaturverzeichnis}

Alexander, J. A./ Weiner, B. J. (1998): The Adoption of the Corporate Governance Model by Nonprofit Organizations, in: Nonprofit Management and Leadership, Vol. 8/No. 8, 223-242.

Benjamin, L. M. (2010): Funders as principals: Performance measurement in philanthropic relationship, in: Nonprofit Management and Leadership, Vol. 20/No. 4, 383-403.

Blühdorn, I. (2007): The third transformation of democracy: on the efficient management of late-modern complexity, in: Blühdorn, I./ Jun, U. (Eds.): Economic Efficiency - Democratic Empowerment. Contested Modernisation in Britain and Germany, Plymouth: Lexington Books, 299-331.

Bozzini, E./ Enjolras, B. (Eds.) (2011): Governing ambiguities, Baden-Baden: Nomos Verlag.

Bradshaw, P. (2009): A contingency approach to nonprofit governance, in: Nonprofit Management and Leadership, Vol. 20/No. 1, 61-81.

Budäus, D. (Hrsg.) (2005): Governance von Profit- und Nonprofit-Organisationen in gesellschaftlicher Verantwortung, Wiesbaden: Gabler Edition Wissenschaft.

Cornforth, C. (2011): Nonprofit Governance Research: Limitations of the Focus on Boards and Suggestions for New Directions, in: Nonprofit and Voluntary Sector Quarterly, online veröffentlicht am 17. November 2011, URL: http://nvs.sagepub.com/content/early/ 2011/11/14/0899764011427959 (Stand: 13. März 2012).

Cornforth, C. (2003): Introduction the Changing Context of Governance-emerging Issues and Paradoxes, in: Comforth, C. (Eds.): The Governance of Public and Non-profit Organisations, London: Routledge, 1-20.

Crouch, C. (2004): Post-democracy, Cambridge et al.: Polity.

Enjolras, B. (2009): A governance-structure approach to voluntary organizations, in: Nonprofit and Voluntary Sector Quarterly, Vol. 38/No. 5, 761-783.

Evans, B./ Richmond, T./ Shields, J. (2005): Structuring Neoliberal Governance: The Nonprofit Sector, Emerging New Modes of Control and the Marketisation of Service Delivery, in: Policy \& Society, Vol. 24/No. 1, 73-97.

Foucault, M. (1983): Der Wille zum Wissen: Sexualität und Wahrheit 1, Frankfurt am Main: Suhrkamp.

Foucault, M. (2000): Die ,Gouvernementalität' (Vorlesung am Collège de France im Studienjahr 1977-78), in: Bröckling, U./ Krasmann, S./Lemke, T. (Hrsg.): Gouvernementalität der Gegenwart: Studien zur Ökonomisierung des Sozialen, Frankfurt am Main: Suhrkamp, 41-67.

Foucault, M. (2007 [1975]): Die Anormalen. Vorlesungen am Collège de France (1974-1975), Frankfurt am Main: Suhrkamp Verlag. 
Freeman, R. E. (1984): Strategic Management: A Stakeholder Approach, London: Pitman Books.

Hugues, O. (2010): Does governance exist?, in: Osborne, S. B. (Eds.): The new public governance: Emerging perspectives on the theory and practice of public governance, London: Routledge, 87-104.

Hwang, H./ Powell, W. W. (2009) The Rationalization of Charity: The Influences of Professionalism in the Nonprofit Sector, in: Administrative Science Quarterly, Vol. 54/No. 2, 268-298.

Jensen, M.C./ Meckling, W.H. (1976): Theory of the firm: Managerial behavior, agency costs and ownership structure, in: Journal of financial economics, Vol. 3/No. 4, 305-360.

LeRoux, K. (2009): Paternalistic or Participatory Governance? Examining Opportunities for Client Participation in Nonprofit Social Service Organizations, in: Public Administration Review, Vol. 69/No. 3, 504-517.

Lynn Jr., L. E./ Heinrich, C. J./ Hill, C. J. (2000): Studying governance and public management: Why? How?, in: Heinrich, C. J./ Lynn Jr., L. E. (Eds.): Governance and performance: New perspectives, Washington, DC: Georgetown University Press, 1-33.

Maier, F./ Meyer, M. (2011): Managerialism and Beyond: Discourses of Civil Society Organization and Their Governance Implications, in: Voluntas: International Journal of Voluntary and Nonprofit Organizations, Vol. 22/No. 4, 731-756.

Miller-Millesen, J. L. (2003): Understanding the behavior of nonprofit boards of directors: A theory-based approach, in: Nonprofit and Voluntary Sector Quarterly, Vol. 32/No. 4, 521-547.

Muth, M./ Donaldson, L. (1998): Stewardship theory and board structure: A contingency approach, in: Corporate Governance: An International Review, Vol. 6/No. 1, 5-28.

Ostrower, F./ Stone, M. M. (2010): Moving governance research forward: a contingency-based framework and data application, in: Nonprofit and Voluntary Sector Quarterly, Vol. 39/No. 5, 901-924.

Pfeffer, J./ Salancik, G. R. (2003): The external control of organizations: A resource dependence perspective, Stanford: Stanford Business Books.

Rosenau, J. N./ Czempiel, E. O. (Eds.) (1992): Governance without government: order and change in world politics, Cambridge et al.: Cambridge University Press.

Rhodes, R. A. W. (1997): Understanding governance: Policy networks, governance, reflexivity, and accountability, Buckingham, PA: Open University Press.

Seckinelgin, H. (2004): Who can help people with HIV/AIDS in Africa? Governance of HIV/AIDS and Civil Society, in: Voluntas: International Journal of Voluntary and Nonprofit Organizations, Vol. 15/No. 3, 287-304.

Siebart, P. (2006): Corporate Governance von Nonprofit-Organisationen. Ausgewählte Aspekte der Organisation und Führung, Bern: Haupt.

Speckbacher, G. (2008): Nonprofit versus corporate governance: An economic approach, in: Nonprofit Management and Leadership, Vol. 18/No. 3, 295-320.

Steen-Johnsen, K./ Eynaud, P./ Wijkström, F. (2011): On Civil Society Governance: An Emergent Research Field, in: Voluntas: International Journal of Voluntary and Nonprofit Organizations, Vol. 22/No. 4, 555-565.

Stone, M. M./ Ostrower, F. (2007): Acting in the Public Interest? Another Look at Research on Nonprofit Governance, in: Nonprofit and Voluntary Sector Quarterly, Vol. 36/No. 3, 416-438.

Villadsen, K. (2009): The 'Human' Touch: Voluntary organizations as rescuers of social policy?, in: Public Management Review, Vol. 11/No. 2, 217-234. 
von Schnurbein, G. (2008): Nonprofit Governance in Verbänden: Theorie und Umsetzung am Beispiel von Schweizer Wirtschaftsverbänden, Bern: Haupt.

von Schnurbein, G./ Stöckli, S. (2010): Die Gestaltung von Nonprofit Governance Kodizes in Deutschland und der Schweiz - eine komparative Inhaltsanalyse, in: Die Betriebswirtschaft, Jg. 70/Heft 6, 493-509.

Zimmer, A./ Freise, M. (2007): Bringing society back in: civil society, social capital and the third sector, in: Maloney, W. A./ Deth, J. W. van (Eds.): Civil Society and Goverance in Europe, Cheltenham: Edward Elgar, 19-42. 